\title{
On uniqueness of limit cycles in general Bogdanov-Takens bifurcation
}

\author{
Maoan Han ${ }^{1 *}$, Jaume Llibre ${ }^{2 \dagger}$ and Junmin Yang ${ }^{3 \ddagger}$ \\ ${ }^{1}$ Department of Mathematics, Shanghai Normal University, 100 Guilin Road \\ Shanghai 200234, China \\ ${ }^{2}$ Departament de Matemàtiques, Universitat Autònoma de Barcelona, 08193 Bellaterra \\ Barcelona, Catalonia, Spain \\ ${ }^{3}$ College of Mathematics and Information Science, Hebei Normal University \\ Shijiazhuang, Hebei 050024, China
}

\begin{abstract}
In this paper we present a complete study to the well-known BogdanovTakens bifurcation and give a rigorous proof for the uniqueness of limit cycles.
\end{abstract}

\section{Introduction}

General theory of Bogdanov-Takens bifurcation is well-known. It was established by Bogdanov in [1] and then introduced in many books, see [2, 3, 4, 5, 13] and [9, 10, 20, 21]. However, to our knowledge, we have not found a really satisfactory and complete study in current works in English. For example, the uniqueness of limit cycles in homoclinic bifurcation was not considered in all of these works in English. The problem was first found and solved in the paper [14]. One can find a relatively complete study on it in books $[9,10,20,21]$ with different details. These books are all in Chinese. The aim of

\footnotetext{
*Corresponding author. mahan@shnu.edu.cn

†jllibre@mat.uab.cat

†jmyang@hebtu.edu.cn
} 
this paper is to provide a complete study with all details on the well-known BogdanovTakens bifurcation.

We organize the paper as follows. In section 2 we state some preliminary theorems which are useful in studying Bogdanov-Takens bifurcation. The preliminary theorems on Hopf and homoclinic bifurcations were obtained after the paper of Bogdanov [1]. In section 3 we study Bogdanov-Takens bifurcation in detail. In section 4 we provide some examples to show that our proof to Bogdanov-Takens bifurcation, especially the proof to the uniqueness of limit cycles in Hopf and homoclinic bifurcations, is really necessary.

\section{Preliminary theorems: a short survey}

Consider a $C^{\infty}$ system on the plane of the form

$$
\begin{aligned}
& \dot{x}=f(x, y)+\varepsilon f_{0}(x, y, \varepsilon, \delta), \\
& \dot{y}=g(x, y)+\varepsilon g_{0}(x, y, \varepsilon, \delta),
\end{aligned}
$$

where $f, g, f_{0}, g_{0}$ are $C^{\infty}$ functions, $\varepsilon$ is small, and $\delta \in G \subset \mathbb{R}^{n}$ with $G$ compact and $n \geq 1$. Suppose that for $\varepsilon=0$ system (2.1) has a $C^{\infty}$ first integral $H(x, y)$, such that the equation $H(x, y)=h$ defines a closed orbit $L_{h}$ for $h \in J$, where $J=(\alpha, \beta)$ is an open interval. Here, we give a short survey on general theory of limit cycle bifurcation occurred in system (2.1). For simplicity, we suppose that the limit of $L_{h}$ as $h \rightarrow \alpha$ exists and is an elementary center of $\left.(2.1)\right|_{\varepsilon=0}$, denoted by $C$, while the limit of $L_{h}$ as $h \rightarrow \beta$ exists and is a homoclinic loop of the unperturbed system, denoted by $L$. The loop $L$ passes through a hyperbolic saddle $S$. Then $C$ and $L$ together form the boundary of the family $\left\{L_{h}\right\}$. To state some results on the number of limit cycles bifurcated from the family and its boundary, introduce the function below

$$
M(h, \delta)=\left.\oint_{L_{h}}\left(f g_{0}-g f_{0}\right) e^{-\int_{0}^{t}\left(f_{x}+g_{y}\right) d \tau}\right|_{\varepsilon=0} d t, h \in J .
$$

First, for $h \in J$, we have the following result on the limit cycle bifurcation from the period annulus defined by $\left\{L_{h}\right\}$ which can be found in $[18,12]$.

Theorem 2.1. Let $M(h, \delta)$ be the function defined by (2.2). Then

(i) For $h_{0} \in J$, a necessary condition for $L_{h_{0}}$ to generate a limit cycle is $M\left(h_{0}, \delta_{0}\right)=0$ for some $\delta_{0} \in G$.

(ii) If

$$
M\left(h_{0}, \delta_{0}\right)=0, \quad \frac{\partial M}{\partial h}\left(h_{0}, \delta_{0}\right) \neq 0,
$$


then there exists a constant $\varepsilon_{0}>0$ such that for $0<|\varepsilon|<\varepsilon_{0}$ and $\left|\delta-\delta_{0}\right|<\varepsilon_{0}$ system (2.1) has a unique limit cycle near $L_{h_{0}}$.

(iii) If

$$
\frac{\partial^{k} M}{\partial h^{k}}\left(h_{0}, \delta_{0}\right) \neq 0, \quad \frac{\partial^{j} M}{\partial h^{j}}\left(h_{0}, \delta_{0}\right)=0, \quad j=0,1, \cdots, k-1
$$

for some integer $k \geq 1$, then there exists a constant $\varepsilon_{0}>0$ such that for $0<|\varepsilon|<\varepsilon_{0}$ and $\left|\delta-\delta_{0}\right|<\varepsilon_{0}$ system (2.1) has at most $k$ limit cycles near $L_{h_{0}}$.

According to [18], the above theorem was proved by L. Pontryagin in 1934 in the case $f_{x}+g_{y} \equiv 0$. The above general case was obtained by Urabe in 1954 , and X. Chen in 1963 respectively.

Now we consider a Hopf bifurcation near the center $C$. Without loss of generality, we assume that $H(0,0)=0$, and that for $x^{2}+y^{2}$ small we have

$$
\begin{aligned}
& f(x, y)+\varepsilon f_{0}(x, y, \varepsilon, \delta)=a(\varepsilon, \delta) x-b(\varepsilon, \delta) y+O\left(x^{2}+y^{2}\right) \\
& g(x, y)+\varepsilon g_{0}(x, y, \varepsilon, \delta)=b(\varepsilon, \delta) x+a(\varepsilon, \delta) y+O\left(x^{2}+y^{2}\right) .
\end{aligned}
$$

In this case the center $C$ has been moved to the origin. By introducing the polar coordinates $x=r \cos \theta, y=r \sin \theta$ one can obtain from (2.1) a $C^{\infty}$ periodic equation of the form

$$
\frac{d r}{d \theta}=R(\theta, r, \varepsilon, \delta)
$$

Let $r\left(\theta, x_{0}, \varepsilon, \delta\right)$ denote the solution of this equation satisfying $r(0)=x_{0}$. Define

$$
\Delta\left(x_{0}, \varepsilon, \delta\right)=r\left(2 \pi, x_{0}, \varepsilon, \delta\right)-x_{0} .
$$

Note that $\Delta\left(x_{0}, 0, \delta\right)=0$ for $\left|x_{0}\right|$ small. We can write for $\left|x_{0}\right|$ small

$$
\Delta\left(x_{0}, \varepsilon, \delta\right)=\varepsilon \sum_{i \geq 1} \Delta_{i}(\varepsilon, \delta) x_{0}^{i}
$$

It is known that the right-lower index $i$ of the first nonzero coefficient $\Delta_{i}$ must be odd.

For (2.3) we have

$$
\Delta_{1}(\varepsilon, \delta)=\frac{1}{\varepsilon}\left[e^{\frac{2 \pi a}{b}}-1\right], \quad \Delta_{1}(0, \delta)=\frac{2 \pi}{\bar{b}_{0}} \frac{\partial a}{\partial \varepsilon}(0, \delta),
$$

where $\bar{b}_{0}=b(0, \delta)$.

The following theorem was proved in $[10,6]$.

Theorem 2.2 (Hopf bifurcation) Let (2.4) hold. Then

(1) If

$$
\Delta_{2 k+1}\left(0, \delta_{0}\right) \neq 0, \quad \Delta_{i}\left(0, \delta_{0}\right)=0, \quad i=1, \cdots, 2 k
$$


for some $\delta_{0} \in G, k \geq 0$, then there exist $\varepsilon_{0}>0$ and a neighborhood $U$ of the origin such that system (2.1) has at most $k$ limit cycles in $U$ for $0<|\varepsilon|<\varepsilon_{0}$ and $\left|\delta-\delta_{0}\right|<\varepsilon_{0}$.

(2) Let $Q(h)=(q(h), 0)$ denote the intersection point of $L_{h}$ with the positive $x$-axis with $q(h)>0$, then

$$
\Delta_{3}(0, \delta)=-\operatorname{sgn}\left(b_{0}\right) \lim _{h \rightarrow 0} \frac{M(h, \delta)}{q^{3} \sqrt{f^{2}(Q)+g^{2}(Q)}}
$$

provided $\Delta_{1}(0, \delta)=0$. In general, we have

$$
\Delta_{2 k+1}(0, \delta)=-\operatorname{sgn}\left(b_{0}\right) \lim _{h \rightarrow 0} \frac{M(h, \delta)}{q^{2 k+1} \sqrt{f^{2}(Q)+g^{2}(Q)}},
$$

provided $\Delta_{i}(0, \delta)=0, i=1, \cdots, 2 k-1$.

(3) If system (2.1) is analytic, $(f, g)= \pm\left(-H_{y}, H_{x}\right)$ and $M\left(h, \delta_{0}\right) \not \equiv 0$ for some $\delta_{0} \in G$, then for some $k \geq 0$ and $N_{k} \neq 0$

$$
M\left(h, \delta_{0}\right)=N_{k} h^{k+1}+O\left(h^{k+\frac{3}{2}}\right) .
$$

In this case, $\Delta_{i}\left(0, \delta_{0}\right)=0, i=1, \cdots, 2 k-1$, and $\Delta_{2 k+1}\left(0, \delta_{0}\right)=K_{k} N_{k}$ for some constant $K_{k} \neq 0$. For example, when $H(x, y)=K\left(x^{2}+y^{2}\right)+o\left(x^{2}+y^{2}\right)$ we have $\Delta_{3}\left(0, \delta_{0}\right)= \pm \frac{K}{2} N_{1}$.

On the uniqueness of limit cycles in a Hopf bifurcation we have the following corollary coming from the above theorem.

Corollary 2.1. If $\frac{\partial a}{\partial \varepsilon}\left(0, \delta_{0}\right)=0$ and $\Delta_{3}\left(0, \delta_{0}\right) \neq 0$, then there exists $\varepsilon_{0}>0$ and a neighborhood $U$ of the origin such that for $0<|\varepsilon|<\varepsilon_{0}$ and $\left|\delta-\delta_{0}\right|<\varepsilon_{0}$, system (2.1) has a unique limit cycle in $U$ if and only if $\mu \varepsilon a(\varepsilon, \delta)<0$ and $\mu=\operatorname{sgn}\left(\bar{b}_{0} \Delta_{3}\left(0, \delta_{0}\right)\right)$.

As usual, the set defined by the equation $a(\varepsilon, \delta)=0$ on the $(\varepsilon, \delta)$ space is called a Hopf bifurcation curve.

It was proved in [7] that as $f_{x}+g_{y}=0$, then $M$ is $C^{\infty}$ at $h=0$. Moreover, $M$ is analytic at $h=0$ if system (2.1) is analytic. Therefore in the case of $f_{x}+g_{y}=0$ for $h$ small we can write

$$
M(h, \delta)=b_{0}(\delta) h+b_{1}(\delta) h^{2}+b_{2}(\delta) h^{3}+\cdots
$$

The formulas for $b_{0}, b_{1}, b_{2}$ can be found in [8]. For example, if

$$
\begin{gathered}
(f(x, y), g(x, y))=\left(H_{y}(x, y),-H_{x}(x, y)\right), \\
H(x, y)=h_{c}+\frac{1}{2}\left(\left(x-x_{c}\right)^{2}+\left(y-y_{c}\right)^{2}\right)+\sum_{i+j \geq 3} h_{i j}\left(x-x_{c}\right)^{i}\left(y-y_{c}\right)^{j},
\end{gathered}
$$




$$
\left.\left(f_{0 x}+g_{0 y}\right)\right|_{\varepsilon=0}=\sum_{i+j \geq 0} c_{i j}\left(x-x_{c}\right)^{i}\left(y-y_{c}\right)^{j},
$$

then for $0<h-h_{c} \ll 1$ we have

$$
M(h, \delta)=\left.\oint_{L_{h}}\left(g_{0} d x-f_{0} d y\right)\right|_{\varepsilon=0}=b_{0}(\delta)\left(h-h_{c}\right)+b_{1}(\delta)\left(h-h_{c}\right)^{2}+\cdots,
$$

where

$$
b_{0}=2 \pi c_{00}, b_{1}=-c_{10} \pi\left(h_{12}+3 h_{30}\right)-c_{01} \pi\left(h_{21}+3 h_{03}\right)+c_{20} \pi+c_{02} \pi .
$$

Hence, by Theorem 2.1 a necessary condition in order that the center $C\left(x_{c}, y_{c}\right)$ generates a limit cycle is $c_{00}\left(\delta_{0}\right)=0$ for some $\delta_{0} \in G$. If $c_{00}\left(\delta_{0}\right)=0$ and $b_{1}\left(\delta_{0}\right) \neq 0$ for some $\delta_{0} \in G$, then there exist a constant $\varepsilon_{0}>0$ and a neighborhood $U$ of the center $C$ such that system (2.1) has at most one limit cycle in $U$ for $0<|\varepsilon|<\varepsilon_{0}$ and $\left|\delta-\delta_{0}\right|<\varepsilon_{0}$. Further, note that in this case $\bar{b}_{0}=-1, \Delta_{3}\left(0, \delta_{0}\right)=-b_{1} / 4$ and $a(\varepsilon, \delta)=\varepsilon c_{00} / 2+O\left(\varepsilon^{2}\right)$. If $\delta \in \mathbb{R}$ and $c_{00}^{\prime}\left(\delta_{0}\right)=\frac{d c_{00}}{d \delta}\left(\delta_{0}\right) \neq 0$, then there exists a function $\varphi_{c}(\varepsilon)=\delta_{0}+O(\varepsilon)$, corresponding to the Hopf bifurcation, such that for $0<|\varepsilon|<\varepsilon_{0}$ and $\left|\delta-\delta_{0}\right|<\varepsilon_{0}$, system (2.1) has a unique limit cycle near the center $C$ if and only if

$$
b_{1}\left(\delta_{0}\right) c_{00}^{\prime}\left(\delta_{0}\right)\left(\delta-\varphi_{c}(\varepsilon)\right)<0
$$

Next, we consider the homoclinic bifurcation. Let $L$ be a homoclinic loop defined by the equation $H(x, y)=\beta$. The loop intersects the $x$-axis transversally at a point $A_{0}$, which is not singular. Then for $h$ near $\beta$ the periodic orbit $L_{h}$ intersects the $x$-axis at a point $Q(h)=(q(h), 0)$ satisfying $Q(h) \rightarrow A_{0}$ as $h \rightarrow \beta$. For $|\varepsilon|$ small system (2.1) has a unique saddle $\tilde{S}\left(x_{s}(\varepsilon, \delta), y_{s}(\varepsilon, \delta)\right)$ near the saddle $S$ on $L$. By the normal form theory there exists a polynomial change of variables which carries system (2.1), near $\tilde{S}$, into a system of the form

$$
\begin{aligned}
& \dot{u}=\lambda_{1}(\varepsilon, \delta) u+a_{1}(\varepsilon, \delta) u^{2} v+O\left(u^{3} v^{2}\right) \\
& \dot{v}=-\lambda_{2}(\varepsilon, \delta) v-b_{1}(\varepsilon, \delta) u v^{2}+O\left(u^{2} v^{3}\right)
\end{aligned}
$$

where $(u, v)=\left(x-x_{s}, y-y_{s}\right)$.

Introduce the following four quantities

$$
\begin{aligned}
& c_{0}(\delta)=M(\beta, \delta), \\
& c_{1}(\delta)=\left.\frac{\partial}{\partial \varepsilon}\left(\lambda_{1}-\lambda_{2}\right)\right|_{\varepsilon=0}=\left.\left(f_{0 x}+g_{0 y}\right)\right|_{\varepsilon=0,(x, y)=S}, \\
& c_{2}(\delta)=-\frac{1}{\sqrt{f^{2}\left(A_{0}\right)+g^{2}\left(A_{0}\right)}} \lim _{h \rightarrow \beta} \frac{M(h, \delta)}{d\left(Q(h), A_{0}\right)}, \\
& c_{3}(\delta)=\left.\frac{\partial}{\partial \varepsilon}\left(a_{1}-b_{1}\right)\right|_{\varepsilon=0} .
\end{aligned}
$$


On the existence of at most one, two, or three limit cycles we have the following theorem which was proved in [10].

Theorem 2.3 (Homoclinic bifurcation). Let $M(h, \delta)$ be the function defined in (2.2). Then the following statements hold.

(1) For $|\varepsilon|$ small a necessary condition in order that system (2.1) has a limit cycle near $L$ is $c_{0}\left(\delta_{0}\right)=0$ for some $\delta_{0} \in G$.

(2) If $c_{0}\left(\delta_{0}\right)=0$ and $c_{1}\left(\delta_{0}\right) \neq 0$, then system (2.1) has at most one limit cycle near $L$ for $|\varepsilon|$ and $\left|\delta-\delta_{0}\right|$ both sufficiently small.

(3) If $c_{0}\left(\delta_{0}\right)=c_{1}\left(\delta_{0}\right)=0$, then $c_{2}\left(\delta_{0}\right)$ is finite, and when $c_{2}\left(\delta_{0}\right) \neq 0$ system $(2.1)$ has at most two limit cycles near $L$ for $|\varepsilon|$ and $\left|\delta-\delta_{0}\right|$ both sufficiently small.

(4) If $c_{0}\left(\delta_{0}\right)=c_{1}\left(\delta_{0}\right)=c_{2}\left(\delta_{0}\right)=0$ and $c_{3}\left(\delta_{0}\right) \neq 0$, then system (2.1) has at most three limit cycles near $L$ for $|\varepsilon|$ and $\left|\delta-\delta_{0}\right|$ both sufficiently small.

(5) If $f_{x}+g_{y} \equiv 0$ and $c_{0}\left(\delta_{0}\right)=c_{1}\left(\delta_{0}\right)=0$, then

$$
c_{2}\left(\delta_{0}\right)=\left.\oint_{L}\left(f_{0_{x}}+g_{0_{y}}\right)\right|_{(\varepsilon, \delta)=\left(0, \delta_{0}\right)} d t .
$$

For the existence of a unique limit cycle the authors of [14] obtained the result below. A proof can be found in $[10,8]$ also.

Theorem 2.4. Let $\delta \in \mathbb{R}$ and let $c_{0}\left(\delta_{0}\right)=0$ for some $\delta_{0} \in G$.

(1) If $\frac{\partial c_{0}}{\partial \delta}\left(\delta_{0}\right) \neq 0$, then there exists a unique differentiable function $\delta^{*}(\varepsilon)=\delta_{0}+O(\varepsilon)$ such that for $|\varepsilon| \neq 0$ and $\left|\delta-\delta_{0}\right|$ both small system (2.1) has a homoclinic loop near $L$ if and and only if $\delta=\delta^{*}(\varepsilon)$.

(2) If either $c_{1}\left(\delta_{0}\right) \neq 0$, or $\lambda_{1}(\varepsilon, \delta) \equiv \lambda_{2}(\varepsilon, \delta)$ and $c_{2}\left(\delta_{0}\right) \neq 0$, then for $|\varepsilon| \neq 0$ and $\left|\delta-\delta_{0}\right|$ both small system (2.1) has at most one limit cycle near L. If additionally $\frac{\partial c_{0}}{\partial \delta}\left(\delta_{0}\right) \neq 0$, then the limit cycle exists if and only if $\mu\left(\delta-\delta^{*}(\varepsilon)\right)>0$, where

$$
\mu=\left\{\begin{array}{l}
\operatorname{sgn}\left(\frac{\partial c_{0}}{\partial \delta}\left(\delta_{0}\right) c_{1}\left(\delta_{0}\right)\right), \text { if } c_{1}\left(\delta_{0}\right) \neq 0, \\
\operatorname{sgn}\left(\frac{\partial c_{0}}{\partial \delta}\left(\delta_{0}\right) c_{2}\left(\delta_{0}\right)\right), \text { if } c_{1}\left(\delta_{0}\right)=0, c_{2}\left(\delta_{0}\right) \neq 0 .
\end{array}\right.
$$

As usual, the curve defined by $\delta=\delta^{*}(\varepsilon)$ is called a homoclinic bifurcation curve of system (2.1).

An useful tool in the proof of the above theorem is a result on the measure of the distance between the two separatrice near $L$ obtained in 1963 by V. Melnikov in [15]. 
If $f_{x}+g_{y} \equiv 0$ and system $(2.1)_{\varepsilon=0}$ is Hamiltonian, then we have for the function $M(h, \delta)$ the following expansion

$$
M(h, \delta)=\sum_{k \geq 0}\left[c_{2 k}(\delta)(h-\beta)^{k}+c_{2 k+1}(\delta)(h-\beta)^{k+1} \ln |h-\beta|\right] .
$$

It was proved in [16] that system (2.1) has at most $k$ limit cycles near $L$ for $|\varepsilon| \neq 0$ and $\left|\delta-\delta_{0}\right|$ both small if $c_{j}\left(\delta_{0}\right)=0, j=0, \cdots, k-1$ and $c_{k}\left(\delta_{0}\right) \neq 0$.

For the formulas of $c_{0}, c_{1}, c_{2}$ and $c_{3}$ see [11]. For the formulas with more coefficients see the recent work [17].

\section{Bogdanov-Takens bifurcation}

In this section we use the theorems listed in section 2 to study the Bogdanov-Takens bifurcation. The problem was studied by Bogdanov in 1976 and Takens in 1974 separately. Consider a $C^{\infty}$ planar differential system of the form

$$
\dot{x}=P(x, y, a), \quad \dot{y}=Q(x, y, a),
$$

where $a \in \mathbb{R}^{2}$ for simplicity. Let system (3.1) have a nilpotent singular point $\left(x_{0}, y_{0}\right)$ for a value of $a$, say $a_{0}$. Without loss of generality we can suppose that $\left(x_{0}, y_{0}\right)=(0,0), a_{0}=0$ and that for $a=0$ we have

$$
\left.\frac{\partial(P, Q)}{\partial(x, y)}\right|_{(x, y)=(0,0)}=\left(\begin{array}{ll}
0 & 1 \\
0 & 0
\end{array}\right) .
$$

Then it is easy to see that for $|a|$ small system (3.1) can be transformed into

$$
\begin{aligned}
& \dot{x}=y, \\
& \dot{y}=g(x, a)+f(x, a) y+O\left(y^{2}\right),
\end{aligned}
$$

near the origin, where

$$
\begin{aligned}
g(x, a) & =g_{0}(a)+g_{1}(a) x+g_{2}(a) x^{2}+O\left(x^{3}\right), \\
f(x, a) & =f_{0}(a)+f_{1}(a) x+O\left(x^{2}\right), \\
g_{0}(0) & =g_{1}(0)=f_{0}(0)=0 .
\end{aligned}
$$

For the sake of simplicity assume $f_{1}(0) g_{2}(0) \neq 0$. Since $g_{2}(0) \neq 0$ there exists a unique function $x_{0}(a)=O(a)$ such that $g_{x}\left(x_{0}(a), a\right)=0$. Then for $\left|x-x_{0}(a)\right|$ small we have

$$
g(x, a)=g\left(x_{0}(a), a\right)+\left(g_{2}(0)+O(a)\right)\left(x-x_{0}(a)\right)^{2}+O\left(\left|x-x_{0}(a)\right|^{3}\right),
$$




$$
f(x, a)=\bar{f}_{0}(a)+\left(f_{1}(0)+O(a)\right)\left(x-x_{0}(a)\right)+O\left(\left|x-x_{0}(a)\right|^{2}\right),
$$

where

$$
g\left(x_{0}(a), a\right)=g_{0}(a)+O\left(|a|^{2}\right), \bar{f}_{0}(a)=f\left(x_{0}(a), a\right) .
$$

Furthermore suppose

$$
a=\left(a_{1}, a_{2}\right) \in R^{2},\left.\quad \operatorname{det} \frac{\partial\left(g_{0}, \bar{f}_{0}\right)}{\partial\left(a_{1}, a_{2}\right)}\right|_{a=0} \neq 0 .
$$

This nondegenerate condition ensures that the change of parameters

$$
\mu_{1}=g\left(x_{0}(a), a\right), \quad \mu_{2}=f\left(x_{0}(a), a\right)
$$

has a unique inverse $a=a^{*}\left(\mu_{1}, \mu_{2}\right)$. Then setting $u=x-x_{0}(a)$ and using $x$ again for $u$ from (3.2) under the assumption (3.3) we have

$$
\begin{aligned}
& \dot{x}=y, \\
& \dot{y}=\mu_{1}+\mu_{2} y+g_{2}^{*}(\mu) x^{2}+f_{1}^{*}(\mu) x y+O\left(x^{3}+x^{2} y+y^{2}\right),
\end{aligned}
$$

where $\mu=\left(\mu_{1}, \mu_{2}\right)$ and

$$
g_{2}^{*}(0)=g_{2}(0) \neq 0, \quad f_{1}^{*}(0)=f_{1}(0) \neq 0
$$

By introducing suitable rescaling of the variables $x, y$ and $t$, from system (3.4) we obtain

$$
\begin{aligned}
\dot{x} & =y \equiv P\left(x, y, \mu_{1}, \mu_{2}\right), \\
\dot{y} & =\mu_{1}+\mu_{2} y+x^{2}+x y+O\left(x^{3}+x^{2} y+y^{2}\right) \\
& \equiv Q\left(x, y, \mu_{1}, \mu_{2}\right) .
\end{aligned}
$$

Our aim next is to study the local behavior of system (3.5) near the origin for small values of the parameters $\mu_{1}$ and $\mu_{2}$.

First, noting that

$$
\begin{gathered}
Q\left(x, 0, \mu_{1}, \mu_{2}\right)=\mu_{1}+x^{2}+O\left(x^{3}\right) \\
\frac{\partial(P, Q)}{\partial(x, y)}=\left(\begin{array}{cc}
0 & 1 \\
2 x+y+O\left(x^{2}+x y\right) & \mu_{2}+x+O\left(x^{2}+y\right)
\end{array}\right),
\end{gathered}
$$

one can see that system (3.5) has no singular points for $\mu_{1}>0$, and has the origin as a saddle-node for $\mu_{1}=0$ and $\mu_{2} \neq 0$. For $\mu_{1}<0$ system $(3.5)$ has a saddle $\left(\sqrt{-\mu_{1}}+O\left(\mu_{1}\right), 0\right)$ and a singular point $A\left(\mu_{1}\right)=\left(-\sqrt{-\mu_{1}}+O\left(\mu_{1}\right), 0\right)$ of index +1 . That is, for each $\mu_{2} \neq 0$, system (3.5) undergoes a saddle-node bifurcation at $\mu_{1}=0$. 
The eigen-equation of system (3.5) at $A\left(\mu_{1}\right)$ has the form

$$
\lambda^{2}+\lambda\left(\mu_{2}-\sqrt{-\mu_{1}}+O\left(\mu_{1}\right)\right)+2 \sqrt{-\mu_{1}}+O\left(\mu_{1}\right)=0 .
$$

Hence there exist two functions

$$
\begin{aligned}
& \varphi_{1}\left(\mu_{1}\right)=2 \sqrt{2}\left(-\mu_{1}\right)^{\frac{1}{4}}+\left(-\mu_{1}\right)^{\frac{1}{2}}+O\left(\left|\mu_{1}\right|^{\frac{3}{4}}\right), \\
& \varphi_{2}\left(\mu_{1}\right)=-2 \sqrt{2}\left(-\mu_{1}\right)^{\frac{1}{4}}+\left(-\mu_{1}\right)^{\frac{1}{2}}+O\left(\left|\mu_{1}\right|^{\frac{3}{4}}\right)
\end{aligned}
$$

such that $A\left(\mu_{1}\right)$ is a focus for $\varphi_{2}\left(\mu_{1}\right)<\mu_{2}<\varphi_{1}\left(\mu_{1}\right)$, a node for $\mu_{2}>\varphi_{1}\left(\mu_{1}\right)$ or $\mu_{2}<$ $\varphi_{2}\left(\mu_{1}\right)$, a degenerate node for $\mu_{2}=\varphi_{1}\left(\mu_{1}\right)$ or $\mu_{2}=\varphi_{2}\left(\mu_{1}\right)$. Obviously, there is a function $\varphi\left(\mu_{1}\right)=\sqrt{-\mu_{1}}+O\left(\left|\mu_{1}\right|\right)$, such that $A\left(\mu_{1}\right)$ is stable for $\mu_{2}<\varphi\left(\mu_{1}\right)$, unstable for $\mu_{2}>\varphi\left(\mu_{1}\right)$. This implies that a possible Hopf bifurcation would occur at $A\left(\mu_{1}\right)$ when $\mu_{2}$ varies near $\varphi\left(\mu_{1}\right)$.

To study the problem in detail we introduce the rescaling change of variables

$$
\mu_{2}=-\delta\left|\mu_{1}\right|^{\frac{1}{2}}, \quad x=-u\left|\mu_{1}\right|^{\frac{1}{2}}, \quad y=v\left|\mu_{1}\right|^{\frac{3}{4}}, \quad t=-\tau\left|\mu_{1}\right|^{-\frac{1}{4}},
$$

and then useing $(x, y, t)$ for $(u, v, \tau)$ from system $(3.5)$ we obtain

$$
\begin{aligned}
& \dot{x}=y, \\
& \dot{y}=1-x^{2}+\tilde{\varepsilon}(\delta+x) y+O\left(\tilde{\varepsilon}^{2}\right),
\end{aligned}
$$

where $\tilde{\varepsilon}=\left|\mu_{1}\right|^{\frac{1}{4}}$. Furthermore letting

$$
u=\frac{1}{2}(x+1), \quad v=\frac{1}{2 \sqrt{2}} y, \quad \tau=\sqrt{2} t,
$$

and also using $(x, y, t)$ for $(u, v, \tau)$, we get the system

$$
\begin{aligned}
& \dot{x}=y, \\
& \dot{y}=x(1-x)+\varepsilon(\lambda+x) y+O\left(\varepsilon^{2}\right),
\end{aligned}
$$

where $\lambda=\frac{1}{2}(\delta-1)$ and $\varepsilon=\sqrt{2} \tilde{\varepsilon}$. The unperturbed system of system (3.6) is Hamiltonian with Hamiltonian

$$
H(x, y)=\frac{1}{2} y^{2}-\frac{1}{2} x^{2}+\frac{1}{3} x^{3}
$$

whose level curves define a family of closed orbits $L_{h}$ for $h \in\left(-\frac{1}{6}, 0\right)$. The boundary of this family consists of a center at $(1,0)$ and a homoclinic loop $L_{0}$ passing through a saddle at the origin. The first order Melnikov function of system (3.6) has the form

$$
M(h, \lambda)=\oint_{L_{h}}(\lambda+x) y d x=\lambda I_{0}(h)+I_{1}(h), \quad h \in\left(-\frac{1}{6}, 0\right),
$$


where

$$
I_{j}(h)=\oint_{L_{h}} x^{j} y d x, \quad j=0,1
$$

Direct computations show that $I_{0}(0)=6 / 5$ and $I_{1}(0)=36 / 35$. Thus

$$
M(0, \lambda)=\frac{6}{5} \lambda+\frac{36}{35}=\frac{6}{5}\left(\lambda+\frac{6}{7}\right) .
$$

Then by Theorem 2.4 we immediately have:

Lemma 3.1 There exist a constant $\epsilon_{0}>0$ and a $C^{1}$ function $\lambda^{*}(\varepsilon)=\lambda_{0}+O(\varepsilon)$ with $\lambda_{0}=-6 / 7$, such that for $0<\varepsilon \leqslant \epsilon_{0}$ and $\left|\lambda-\lambda_{0}\right| \leqslant \epsilon_{0}$, system (3.6) has a unique limit cycle (no limit cycle, respectively) near $L_{0}$ if $\lambda<\lambda^{*}(\varepsilon)\left(\lambda>\lambda^{*}(\varepsilon)\right.$, respectively), and has a homoclinic loop near $L_{0}$ if and only if $\lambda=\lambda^{*}(\varepsilon)$. Moreover, the limit cycle or the homoclinic loop is stable.

Rewriting $H(x, y)$ as

$$
H(x, y)=\frac{1}{2}\left(y^{2}+(x-1)^{2}\right)+\frac{1}{3}(x-1)^{3},
$$

then from (2.5) we get

$$
M(h, \lambda)=2 \pi(\lambda+1)\left(h+\frac{1}{6}\right)-\pi\left(h+\frac{1}{6}\right)^{2}+\cdots .
$$

Therefore, from Theorem 2.2 and Corollary 2.1 we obtain:

Lemma 3.2 There exist a constant $\epsilon_{1}>0$ and a $C^{1}$ function $\lambda_{1}^{*}(\varepsilon)=-1+O(\varepsilon)$, such that for $0<\varepsilon \leqslant \epsilon_{1}$ and $|\lambda+1| \leqslant \epsilon_{1}$ system (3.6) has a unique limit cycle near the center $(1,0)$ if and only if $\lambda>\lambda_{1}^{*}(\varepsilon)$. Moreover, the limit cycle is stable when it exists.

Now our next step is to prove that the limit cycle of the above two lemmas exists uniquely for all $\lambda_{1}^{*}(\epsilon)<\lambda<\lambda^{*}(\epsilon)$. For this purpose we rewrite the function $M(h, \lambda)$ as

$$
M(h, \lambda)=I_{0}(h)(\lambda-P(h)), \quad P(h)=-I_{1}(h) / I_{0}(h) .
$$

The following lemma provides the properties of the function $P(h)$ due to Bogdanov [1].

Lemma 3.3 The function $P(h)$ is continuous on $\left[-\frac{1}{6}, 0\right]$, differentiable on $\left[-\frac{1}{6}, 0\right)$ with $P\left(-\frac{1}{6}\right)=-1, P(0)=-\frac{6}{7}, P^{\prime}\left(-\frac{1}{6}\right)=\frac{1}{2}$ and $P^{\prime}(h)>0$ for $\left(-\frac{1}{6}, 0\right)$.

Proof From the proofs of Lemmas 3.1 and 3.2 we have $P(0)=-\frac{6}{7}$ and $P\left(-\frac{1}{6}\right)=-1$. Note that along $L_{h}$ we get

$$
y d y=x d x-x^{2} d x, \frac{\partial y}{\partial h}=\frac{1}{y} .
$$

By writing $I_{1}(h)$ into the form of definite integral in $x$ we find

$$
I_{1}^{\prime}(h)=\oint_{L_{h}} \frac{x d x}{y}=\oint_{L_{h}} \frac{x d x-y d y}{y}=\oint_{L_{h}} \frac{x^{2} d x}{y} .
$$


Then by integration by parts we have

$$
\begin{aligned}
I_{0}(h) & =-\oint_{L_{h}} x d y=-\oint_{L_{h}} \frac{x^{2} d x-x^{3} d x}{y} \\
& =\oint_{L_{h}} \frac{1}{y}\left(3 h+\frac{3}{2} x^{2}-\frac{3}{2} y^{2}-x^{2}\right) d x \\
& =3 h I_{0}^{\prime}(h)+\frac{1}{2} I_{1}^{\prime}(h)-\frac{3}{2} I_{0}(h) .
\end{aligned}
$$

Hence

$$
5 I_{0}=6 h I_{0}^{\prime}+I_{1}^{\prime}
$$

Similarly, integrating by parts the function $I_{1}$ we get

$$
35 I_{1}=6 h I_{0}^{\prime}+6(1+5 h) I_{1}^{\prime}
$$

Solving $I_{0}^{\prime}$ and $I_{1}^{\prime}$ from (3.7) and (3.8) yields to the so-called Picard-Fuchs equations as follows

$$
I_{1}^{\prime}=\frac{1}{1+6 h}\left(7 I_{1}-I_{0}\right), \quad I_{0}^{\prime}=\frac{1}{6 h(1+6 h)}\left[6(1+5 h) I_{0}-7 I_{1}\right]
$$

Therefore we obtain

$$
\frac{d P}{d h}=\frac{-1}{I_{0}^{2}}\left[I_{0} I_{1}^{\prime}-I_{1} I_{0}^{\prime}\right]=\frac{-7 P^{2}+6(2 h-1) P+6 h}{6 h(1+6 h)} .
$$

This means that the function $P=P(h)$ defines an orbit connecting the saddle $\left(-\frac{1}{6},-1\right)$ and the node $\left(0,-\frac{6}{7}\right)$ in the plane of the quadratic system

$$
\begin{aligned}
& \dot{h}=6 h(1+6 h), \\
& \dot{P}=-7 P^{2}+6(2 h-1) P+6 h .
\end{aligned}
$$

It follows that the function $P(h)$ is continuously differentiable on the interval $\left[-\frac{1}{6}, 0\right)$.

Consider the isocline equation

$$
-7 P^{2}+6(2 h-1) P+6 h=0 .
$$

It is a hyperbola with two branches $L_{1}$ and $L_{2}$ with $L_{1}$ lying above $L_{2}$. It is easy to see that $L_{1}$ passes through the points $(0,0)$ and $\left(-\frac{1}{6},-\frac{1}{7}\right)$, while $L_{2}$ passes through the points $\left(0,-\frac{6}{7}\right)$ and $\left(-\frac{1}{6},-1\right)$. Note that for $-\frac{1}{6}<h<0$ we have

$$
\begin{gathered}
\dot{h}=6 h(1+6 h)<0, \\
\dot{P}=-7 P^{2}+6(2 h-1) P+6 h \begin{cases}>0 & \text { between } L_{1} \text { and } L_{2}, \\
=0 & \text { on } L_{1} \text { or } L_{2}, \\
<0 & \text { above } L_{1} \text { or below } L_{2} .\end{cases}
\end{gathered}
$$


It implies that the curve $P=P(h)$ is below $L_{2}$ for $-\frac{1}{6}<h<0$. Thus $P^{\prime}(h)=\frac{d P}{d h}=\frac{\dot{P}}{\dot{h}}>0$. Furthermore the linear variational equation of system $(3.9)$ at the saddle $\left(-\frac{1}{6},-1\right)$ is

$$
\dot{x}=-6 x, \quad \dot{y}=-6 x+6 y,
$$

which has the separatrix $y=\frac{1}{2} x$. Hence $P^{\prime}\left(-\frac{1}{6}\right)=\frac{1}{2}$. This completes the proof.

On the other hand, the variational equation of system (3.9) at the degenerate node $\left(0,-\frac{6}{7}\right)$ has the form

$$
\dot{x}=6 x, \quad \dot{y}=-\frac{30}{7} x+6 y,
$$

from which we get $P^{\prime}(0-0)=\infty$.

Noting that $0 \leq x \leq 3 / 2$ and $|y| \leq 1 / \sqrt{3}$ along the homoclinic loop $L_{0}$ we can suppose in system (3.6) that $-\varepsilon^{*} \leq x \leq 3 / 2+\varepsilon^{*},|y| \leq 1 / \sqrt{3}+\varepsilon^{*}$ and $0<\varepsilon<\varepsilon^{*}$ for a small constant $\varepsilon^{*}>0$. In this case there exists a constant $N>0$ such that the divergence of (3.6) is positive or negative (and hence there is no limit cycle) if $|\lambda|>N$ and $0<\varepsilon<\varepsilon^{*}$. For example, we can take $N=3$ for a suitable $\varepsilon^{*}$ since

$$
|\operatorname{div}(3.6)|=|\varepsilon(\lambda+x+O(\varepsilon))|>\varepsilon(|\lambda|-2+O(\varepsilon)) .
$$

That is, in system (3.6) we can assume $|\lambda| \leq N$ to discuss the existence of limit cycles. With this assumption and applying Lemmas 3.1-3.3 we can prove the following theorem.

Theorem 3.1 There exist a constant $\varepsilon^{*}>0$ and two differentiable functions $\lambda^{*}(\varepsilon)=$ $-\frac{6}{7}+O(\varepsilon)$ and $\lambda_{1}^{*}(\varepsilon)=-1+O(\varepsilon)$ such that for all $0<\varepsilon<\varepsilon^{*}$ and $|\lambda| \leq N$, system (3.6) has

(1) no limit cycles for $\lambda>\lambda^{*}(\varepsilon)$ or $\lambda \leqslant \lambda_{1}^{*}(\varepsilon)$;

(2) a stable homoclinic loop near $L_{0}$ for $\lambda=\lambda^{*}(\varepsilon)$;

(3) a unique (stable) limit cycle for $\lambda_{1}^{*}(\varepsilon)<\lambda<\lambda^{*}(\varepsilon)$.

Proof. Correspondingly we need only to prove the following three statements:

(i) There exists a constant $\varepsilon_{1}^{*}>0$, such that for $0<\varepsilon<\varepsilon_{1}^{*}$ system (3.6) has no limit cycles when either $\lambda^{*}(\varepsilon)<\lambda \leq N$ or $-N \leq \lambda \leq \lambda_{1}^{*}(\varepsilon)$.

(ii) There exists a constant $\varepsilon_{2}^{*}>0$ such that for $0<\varepsilon<\varepsilon_{2}^{*}$ and $\lambda=\lambda^{*}(\varepsilon)$ system (3.6) has a stable homoclinic loop near $L_{0}$.

(iii) There exists a constant $\varepsilon_{3}^{*}>0$ such that for $0<\varepsilon<\varepsilon_{3}^{*}$ and $\lambda_{1}^{*}(\varepsilon)<\lambda<\lambda^{*}(\varepsilon)$ system (3.6) has a unique limit cycle, and it is stable.

When the three statements are proved, we can take $\varepsilon^{*}=\min \left\{\varepsilon_{1}^{*}, \varepsilon_{2}^{*}, \varepsilon_{3}^{*}\right\}$ to complete the proof. 
We shall prove the statements by contradiction. If statement (i) is not true, then there exist sequences $\varepsilon_{n}$ and $\lambda_{n}$ satisfying $\varepsilon_{n}>0$ and $\varepsilon_{n} \rightarrow 0$ as $n \rightarrow \infty$ and $\lambda^{*}\left(\varepsilon_{n}\right)<\lambda_{n} \leq N$, or $-N \leq \lambda_{n} \leq \lambda_{1}^{*}\left(\varepsilon_{n}\right)$ such that for $(\varepsilon, \lambda)=\left(\varepsilon_{n}, \lambda_{n}\right)$ system (3.6) has a limit cycle $L_{n}^{*}$. Note that $\left\{\lambda_{n}\right\}$ and $\left\{L_{n}^{*}\right\}$ are all bounded for all $n$. We can suppose that $\lambda_{n} \rightarrow \lambda^{*}$ and $L_{n}^{*} \rightarrow \Gamma^{*}($ as $n \rightarrow+\infty)$.

Clearly $-\frac{6}{7} \leqslant \lambda^{*} \leqslant N$, or $-N \leq \lambda^{*} \leq-1$, and $\Gamma^{*}$ should be a closed invariant curve of system $(3.6)_{\varepsilon=0}$. This means that $\Gamma^{*}$ is defined by an equation $H(x, y)=h^{*}$ for some $h^{*} \in\left[-\frac{1}{6}, 0\right]$. By Theorems 3.1-3.3 we have necessarily $M\left(h^{*}, \lambda^{*}\right)=0$, or $\lambda^{*}=P\left(h^{*}\right)$, By Lemma 3.3 one obtains $-1 \leqslant \lambda^{*} \leqslant-\frac{6}{7}$. Then by Lemmas 3.1 and 3.2 we have either $\lambda^{*}=-\frac{6}{7}$ and $\Gamma^{*}$ should be the loop $L_{0}$, or $\lambda^{*}=-1$ and $\Gamma^{*}$ should be the center $(1,0)$. In the first case we have $-\frac{6}{7}-\epsilon_{0}<\lambda_{n}<-\frac{6}{7}+\epsilon_{0}$ and $0<\varepsilon_{n}<\epsilon_{0}$ for $n$ sufficiently large, which, together with Lemma 3.1, implies that for $(\varepsilon, \lambda)=\left(\varepsilon_{n}, \lambda_{n}\right)$ system $(3.6)$ has no limit cycles near $L_{0}$ because $\lambda_{n}>\lambda^{*}\left(\varepsilon_{n}\right)$, a contradiction. In the second case a contradiction occurs similarly by Lemma 3.2. Then statement (i) follows.

Statement (ii) is direct from Lemma 3.1.

For statement (iii), first by Poincaré-Bendixson theorem one can see that for $\lambda_{1}^{*}(\varepsilon)<$ $\lambda<\lambda^{*}(\varepsilon)$ with $\varepsilon>0$ small system (3.6) has a limit cycle. We shall prove the uniqueness of the limit cycle by reduction to a contradiction. That is, we suppose that the uniqueness conclusion is not true. Then there exists sequences $\varepsilon_{n}>$ and $\lambda_{n}>0$ satisfying $\varepsilon_{n} \rightarrow 0$ and $\lambda_{1}^{*}\left(\varepsilon_{n}\right)<\lambda_{n}<\lambda^{*}\left(\varepsilon_{n}\right)$ such that for $(\varepsilon, \lambda)=\left(\varepsilon_{n}, \lambda_{n}\right)$ system (3.6) has two limit cycles $L_{n}^{(1)}$ and $L_{n}^{(2)}$. As before we can assume that as $n \rightarrow \infty$ we have $\lambda_{n} \rightarrow \lambda^{*}$ with $-1 \leqslant \lambda^{*} \leqslant-\frac{6}{7}$, and $L_{n}^{(j)} \rightarrow \Gamma_{j}$ for $j=1,2$. Then $\Gamma_{j}$ satisfies $H(x, y)=h_{j}$ for some $h_{j} \in\left[-\frac{1}{6}, 0\right], j=1,2$. Hence, $M\left(h_{j}, \lambda^{*}\right)=0$, and then $\lambda^{*}=P\left(h_{1}\right)=P\left(h_{2}\right)$. Thus $h_{1}=h_{2}$ by Lemma 3.3. Therefore, a contradiction must occur in the same way than in Lemma 3.2 for $h_{1}=-1$, Lemma 3.1 for $h_{1}=-6 / 7$ or Lemma 3.2 and Theorem 1.1 for $h_{1} \in(-1,-6 / 7)$. This ends the proof of Theorem 3.1.

From the above proof, one can see that each one of Lemmas 3.1-3.3 is indispensable and plays its own role in the proof. In other words any one of them is not sufficient to deduce the statements of Theorem 3.1.

Note that $\mu_{2}=-\delta\left|\mu_{1}\right|^{\frac{1}{2}}=-(2 \lambda+1)\left|\mu_{1}\right|^{\frac{1}{2}}, \varepsilon=\sqrt{2}\left|\mu_{1}\right|^{\frac{1}{4}}$. Back to system (3.5) and we have from Theorem 3.1 the following result.

Theorem 3.2 There exist a constant $\epsilon_{0}>0$, a neighborhood $U$ of the origin and two continuous functions $\psi_{1}\left(\mu_{1}\right)=\left(-\mu_{1}\right)^{\frac{1}{2}}+O\left(\left|\mu_{1}\right|^{\frac{3}{4}}\right)$ (corresponding to Hopf bifurcation) and $\psi_{2}\left(\mu_{1}\right)=\frac{5}{7}\left(-\mu_{1}\right)^{\frac{1}{2}}+O\left(\left|\mu_{1}\right|^{\frac{3}{4}}\right)$ (corresponding to homoclinic bifurcation), such that for 
$0 \leqslant-\mu_{1} \leqslant \epsilon_{0}$ and $\left|\mu_{2}\right| \leqslant \epsilon_{0}$, system (3.5) has a unique limit cycle in $U$ if and only if $\psi_{2}\left(\mu_{1}\right)<\mu_{2}<\psi_{1}\left(\mu_{1}\right)$.

\section{Some examples with two limit cycles}

In this section we give two examples which have two limit cycles. However the first order Melnikov function of them has only one nontrivial zero. The first example is a system of Liénard type

$$
\dot{x}=y-\varepsilon\left(\varepsilon x-x^{3}+x^{5}\right), \quad \dot{y}=-x,
$$

where $\varepsilon>0$ is small. The unperturbed system of it is a linear center with the first integral $H(x, y)=x^{2}+y^{2}$. Let $L_{h}$ denote the circle defined by $x^{2}+y^{2}=h$ with $h>0$. Then by (2.2) the first order Melnikov function is

$$
M(h)=\oint_{L_{h}}\left(-x^{3}+x^{5}\right) d y .
$$

Note that $L_{h}$ can be represented as $x=\sqrt{h} \sin t, y=\sqrt{h} \cos t$. A direct computation shows that

$$
\begin{aligned}
M(h) & =\int_{0}^{2 \pi}\left[h^{2} \sin ^{4} t-h^{3} \sin ^{6} t\right] d t \\
& =h^{2}\left[\pi / 2+\frac{1}{4} \int_{0}^{2 \pi} \cos ^{2}(2 t) d t\right]-h^{3}\left[\pi / 4+\frac{3}{8} \int_{0}^{2 \pi} \cos ^{2}(2 t) d t\right] \\
& =\frac{3 \pi}{4} h^{2}(1-5 h / 6),
\end{aligned}
$$

which has a unique simple positive zero $h=6 / 5$. It follows from Theorem 2.1 that for $\varepsilon>0$ small system (4.1) has a unique limit cycle $\Gamma_{1 \varepsilon}$ near the circle $x^{2}+y^{2}=6 / 5$.

Noting that $\operatorname{div}(4.1)=\varepsilon\left(3 x^{2}-5 x^{4}-\varepsilon\right)$, we have

$$
\oint_{\Gamma_{1 \varepsilon}} \operatorname{div}(4.1) \mathrm{dt}=\varepsilon[-9 \pi / 5+\mathrm{O}(\varepsilon)]<0,
$$

for $0<\varepsilon \ll 1$. This implies that the limit cycle $\Gamma_{1 \varepsilon}$ is stable.

On the other hand, the origin is a stable focus for $0<\varepsilon \ll 1$ since

$$
\left.\operatorname{div}(4.1)\right|_{(x, y)=(0,0)}=-\varepsilon^{2} .
$$

Therefore there must have an unstable limit cycle, denoted by $\Gamma_{2 \varepsilon}$, inside $\Gamma_{1 \varepsilon}$. By Theorems 2.1 and 2.2 we obtain that $\Gamma_{1 \varepsilon}$ and $\Gamma_{2 \varepsilon}$ are the only limit cycles of system (4.1). 
From the above discussion one can see that the following system

$$
\dot{x}=y-\varepsilon\left(\varepsilon x-x^{3}\right), \quad \dot{y}=-x,
$$

has a unique limit cycle $\Gamma_{\varepsilon}$ for $0<\varepsilon \ll 1$. However its first order Melnikov function is $M(h)=\frac{3 \pi}{4} h^{2}$, which has no positive zero. This fact implies that for any $h_{0}>0$ there exists $\varepsilon_{0}>0$ such that for $0<\varepsilon<\varepsilon_{0}$ all orbits starting from a point in the region $x^{2}+y^{2}>h_{0}$ are unbounded positively. And, by Theorems 2.1 and 2.2 the limit cycle $\Gamma_{\varepsilon}$ is unique and approaches the origin as $\varepsilon \rightarrow 0$.

Our second example is

$$
\begin{aligned}
& \dot{x}=y+\varepsilon x[\varepsilon+x(a-H(x, y))], \\
& \dot{y}=x-x^{2},
\end{aligned}
$$

where

$$
H(x, y)=\frac{1}{2}\left(y^{2}-x^{2}\right)+\frac{1}{3} x^{3} .
$$

The unperturbed system of system (4.2) has a saddle at the origin, a center at $(1,0)$, and a family of closed orbits given by

$$
L_{h}: H(x, y)=h, \quad h \in\left(-\frac{1}{6}, 0\right) .
$$

The limit of $L_{h}$ as $h \rightarrow 0$, denoted by $L_{0}$, is a homoclinic loop satisfying $H(x, y)=0$. By (2.2) the first order Melnikov function has the form

$$
M(h, a)=I(h)[a-h]
$$

where

$$
I(h)=\oint_{L_{h}}\left(-x^{2}\right) d y=2 \oint_{L_{h}} x y d x .
$$

Clearly $I\left(-\frac{1}{6}\right)=0$. Noting that $y y_{h}=1$ along $L_{h}$, one has

$$
I^{\prime}(h)=\oint_{L_{h}} \frac{2 x}{y} d x=\oint_{L_{h}} 2 x d t>0,
$$

which yields $I(h)>0$ for all $h \in\left(-\frac{1}{6}, 0\right)$. Hence $M(h, a)$ has at most one zero on the interval $\left(-\frac{1}{6}, 0\right)$. From (4.4) one also has $I^{\prime}\left(-\frac{1}{6}\right)=4 \pi$, from which it follows

$$
I(h)=4 \pi\left(h+\frac{1}{6}\right)+O\left(\left|h+\frac{1}{6}\right|^{2}\right),
$$

for $h+\frac{1}{6}>0$ small. Hence by (4.3) we obtain

$$
M(h, a)=4 \pi\left(h+\frac{1}{6}\right)\left[a+\frac{1}{6}-\left(1+K\left(a+\frac{1}{6}\right)\right)\left(h+\frac{1}{6}\right)\right]+O\left(\left|h+\frac{1}{6}\right|^{3}\right),
$$


for $h+\frac{1}{6}>0$ small, where $K$ is a constant. By Corollary 2.1 there exists a function $\varphi_{c}(\varepsilon)=-\frac{1}{6}+O(\varepsilon)$ such that for $\varepsilon+\left|a+\frac{1}{6}\right|$ small system (4.2) has a unique limit cycle, say $\Gamma_{\varepsilon, a}$, in a neighborhood of the center $(1,0)$ if and only if $a>\varphi_{c}(\varepsilon)$. On the $(\varepsilon, a)$-plane the curve $a=\varphi_{c}(\varepsilon)$ defines a Hopf bifurcation curve. Note that by (2.5)

$$
\begin{aligned}
\left.\operatorname{div}(4.2)\right|_{C} & =\varepsilon\left[c_{00}+O(\varepsilon)\right] \\
& =\varepsilon\left[\frac{b_{0}}{2 \pi}+O(\varepsilon)\right] \\
& =\varepsilon[2(a+1 / 6)+O(\varepsilon)],
\end{aligned}
$$

where $C$ denotes the focus of system (4.2) near the center $(1,0)$. Hence

$$
\left.\operatorname{div}(4.2)\right|_{C}>0 \Leftrightarrow a>\varphi_{c}(\varepsilon)
$$

Thus the limit cycle $\Gamma_{\varepsilon, a}$ is stable.

Now we consider the problem: How does the stable limit cycle $\Gamma_{\varepsilon, a}$ vary when the parameter $a$ increases from $\varphi_{c}(\varepsilon)$ ?

To understand the behavior of $\Gamma_{\varepsilon, a}$ as $a$ increases, let $a \in\left(-\frac{1}{6}, 0\right)$. In this case the function $M(h, a)$ has a unique simple zero $h=a$ for $h \in\left(-\frac{1}{6}, 0\right)$. This implies that for $\varepsilon>0$ small system (4.2) has a unique limit cycle near $L_{a}$. Along the limit cycle we have

$$
\begin{aligned}
\oint \operatorname{div}(4.2) d t & =\varepsilon\left[\oint_{L_{a}} x^{2}\left(x-x^{2}\right) d t+O(\varepsilon)\right] \\
& =-\varepsilon[I(a)+O(\varepsilon)] \\
& <0
\end{aligned}
$$

for $\varepsilon>0$ small. Hence the limit cycle is stable. Then it is clear that when $a$ increases from $\varphi_{c}(\varepsilon)$ the stable limit cycle $\Gamma_{\varepsilon, a}$ enlarges constantly, and it approaches to $L_{a}$ as $\varepsilon \rightarrow 0$.

We consider an additional problem: what happens for $a$ near zero?

First noting that $M(0, a)=I(0) a=\frac{72}{35} a$, by Theorem 2.4(1) and its proof there exists a function $\varphi_{h}(\varepsilon)=O(\varepsilon)$ such that (4.2) has a homoclinic loop $\Gamma_{\varepsilon}^{*}$ if and only if $a=\varphi_{h}(\varepsilon)$. Moreover for $a$ near $\varphi_{h}(\varepsilon)$ the orbit behavior of system (4.2) near $L_{0}$ is as shown in Fig.4.1.

For $\left.\operatorname{div}(4.2)\right|_{x=y=0, a=\varphi_{h}(\varepsilon)}=\varepsilon^{2}$ the homoclinic loop $\Gamma_{\varepsilon}^{*}$ is unstable. On the other hand, by (4.5) the focus $C$ is unstable for $\varphi_{c}(\varepsilon)<a \leq \varphi_{h}(\varepsilon)$. Then it follows that system (4.2) has a stable limit cycle, denoted by $\bar{\Gamma}_{\varepsilon}$, inside the loop $\Gamma_{\varepsilon}^{*}$ for $a=\varphi_{h}(\varepsilon)$. In the same way, from Fig. 4.1, the stable limit cycle $\Gamma_{\varepsilon, a}$ obtained before always exists for 


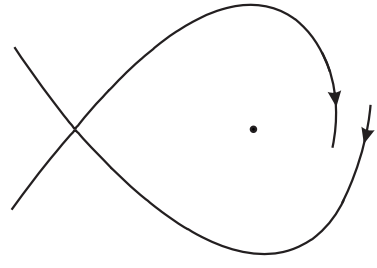

(i) $a<\varphi_{h}(\varepsilon)$

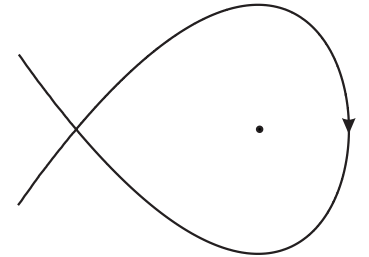

(ii) $a=\varphi_{h}(\varepsilon)$

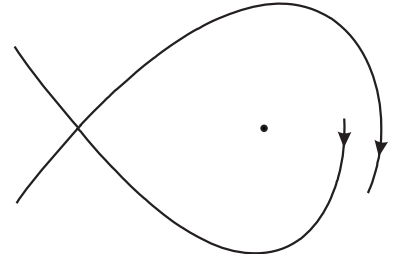

(iii) $a>\varphi_{h}(\varepsilon)$

Fig. 4.1. Phase portraits of system (4.2) near $L_{0}$.

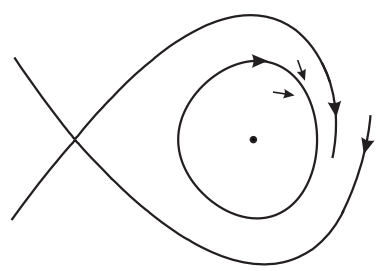

(i) $\varphi_{c}(\varepsilon)<a<\varphi_{h}(\varepsilon)$

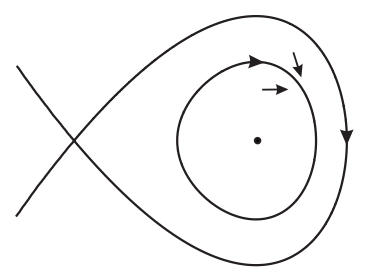

(ii) $a=\varphi_{h}(\varepsilon)$

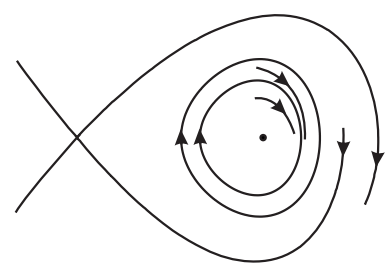

(iii) $0<a-\varphi_{h}(\varepsilon) \ll 1$

Fig. 4.2. Global behavior of system (4.2).

$\varphi_{c}(\varepsilon)<a<\varphi_{h}(\varepsilon)$. Furthermore, for $0<a-\varphi_{h}(\varepsilon) \ll 1$ the homoclinic loop $\Gamma_{\varepsilon}^{*}$ has broken and generated an unstable limit cycle $\Gamma_{\varepsilon, a}^{*}$, see Fig. 4.2.

By Theorem 2.3, $\Gamma_{\varepsilon, a}$ and $\Gamma_{\varepsilon, a}^{*}$ are the only limit cycles of system (4.2).

Finally, for any given constant $a_{0}>0$, we have $M(h, a)>0$ for all $h \in\left[-\frac{1}{6}, 0\right]$ and $a \geq a_{0}$. Hence there exists $\varepsilon_{0}=\varepsilon_{0}\left(a_{0}\right)>0$ such that for $0<\varepsilon<\varepsilon_{0}$ and $a \geq a_{0}$ system (4.2) has no limit cycles. Thus there exists a function $\varphi_{s n}(\varepsilon)=O(\varepsilon)>\varphi_{h}(\varepsilon)$ such that for $a=\varphi_{s n}(\varepsilon)$ system (4.2) has a semi-stable limit cycle which is the limit of $\Gamma_{\varepsilon, a}$ and $\Gamma_{\varepsilon, a}^{*}$ when $a \rightarrow \varphi_{s n}(\varepsilon)$ from left. The function $\varphi_{s n}(\varepsilon)$ corresponds to the saddle-node bifurcation of limit cycles in system (4.2). Therefore we have three bifurcation curves on $(\varepsilon, a)$-plane: Hopf bifurcation $a=\varphi_{c}(\varepsilon)$, homoclinic bifurcation $a=\varphi_{h}(\varepsilon)$ and saddlenode bifurcation of limit cycles $a=\varphi_{s n}(\varepsilon)$. The bifurcation diagram is shown in Fig. 4.3 .

We have seen from examples (4.1) and (4.2) that the study of the uniqueness of limit 


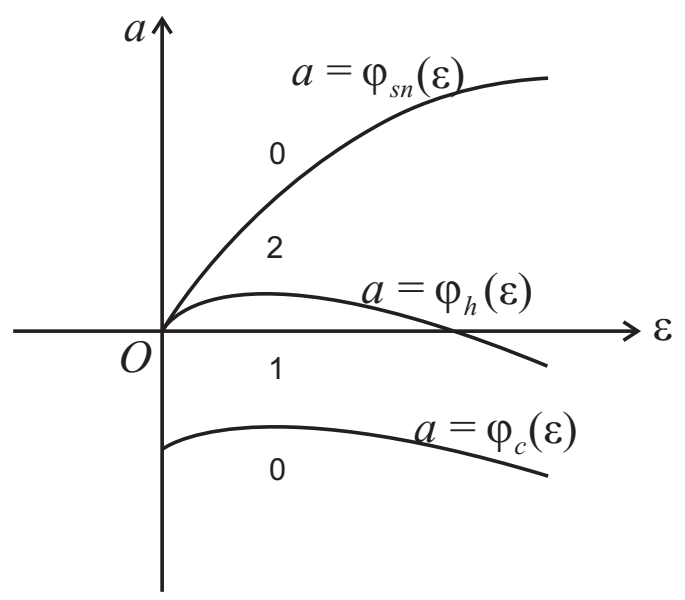

Fig. 4.3. Bifurcation diagram of limit cycles of system (4.2).

cycles near the boundary of the period annulus in the Bogdanov-Takens bifurcation is necessary and non-trivial.

\section{Acknowledgments}

The first and the third authors are partially supported by National Natural Sciences Foundation 11431008, 11771296 and 11571090. The second author is partially supported by the MINECO grants MTM2013-40998-P and MTM2016-77278-P (FEDER) and the AGAUR grant 2014-SGR568.

\section{References}

[1] R.I. Bogdanov, The versal deformation of a singuar point of a vector field on the plane in the case of zero eigenvalues, in "Proceedings of Petrovskii Seminar, Vol. 2, Moscow State University, Moscow, 37-65". In Russian (English translation: Selecta Math. Soviet., 1(1981), 389-421).

[2] J. Carr, Applications of Center Manifold Theory, Springer-Verlag, New York, 1982.

[3] S.N. Chow, J.K. Hale, Methods of Bifurcation Theory, Springer-Verlag, New York, 1996.

[4] S-N. Chow, C. Li, D. Wang, Normal Forms and Bifurcations of Planar Vector Fields (Cambridge University Press), 1994. 
[5] J. Guckenheimer, P. Holmes, Nonlinear Oscillations, Dynamical Systems and Bifurcations of Vector Fields, Springer-Verlag, New York, 1983.

[6] M. Han, Bifurcation of limit cycles and the cusp of order n, Acta Mathematics Sinica, New Series 13(1997), 64-75.

[7] M. Han, On Hopf cyclicity of planar systems, Journal of Mathematical Analysis and Applications 245(2000), 404-422.

[8] M. Han, Bifurcaton Theory of Limit Cycles, Science Press, Beijing, 2012.

[9] M. Han, S. Gu, Theory and Methods of Nonlinear Systems, Science Press, Beijing, 2001 (In Chinese).

[10] M. Han, D. Zhu, Bifurcation Theory of Ordinary Differential Equatipons, Coal Mine Industry Publishing House, Beijing, 1994 (In Chinese).

[11] M. Han, J. Yang, A. Tarta, Y. Gao, Limit cycles near homoclinic and heteroclinic loops, J. Dynamics and Differential Equations 20(2008), 923-944.

[12] J. Li, Hilbert's 16th problem and bifurcations of planar polynomial vector fields. Int. J. Bifurcation Chaos 13(2003), 47-106.

[13] Yuri A. Kuznetsov, Elements of Applied Bifurcation Theory, Springer-Verlag New York, 1995, 1998, 2004.

[14] D. Luo, M. Han, D. Zhu, Uniqueness of limit cycles bifurcated from singular closed orbits (I), Acta Math. Sinica 3(1992), 404-414.

[15] V. K. Melnikov, On the stability of the center for time periodic perturbations, Trans. Moscow Math. Soc., 12(1963), 1-57.

[16] R. Roussarie, On the number of limit cycles which appear by perturbation of separatrix loop of planar vector fields, Bol. Soc. Bras. Mat. 17(1986), 67-101.

[17] Y. Tian, M. Han, Hopf and homoclinic bifurcations for near-Hamiltonian systems, J. Differential Equations 262(2017), 3214-3234.

[18] Y. Ye, et al., Theory of limit cycles, Shanghai Science and Technology Publishing House, Shanghai 1984(In Chinese), Transl. Math. Monographs, Vol. 66, Amer. Math. Soc., Providence RI, 1986. 
[19] Z. Zhang, B. Li, High order Melnikov functions and the problem of uniformity in global bifurcation, Annali di Mat. Pura ed Appl. (IV), vol. CLX(1992), 181-212.

[20] Z. Zhang, C., Li, Z. Zheng, W. Li, Elementary Theory of Bifurcations of Vector Fields (Higher Education Press, Beijing), 1997 (in Chinese).

[21] A. Zhao, M. Li, M. HanFundamental Theory of Differential EquationsScience Press, Beijing2011(In Chinese). 